\title{
Editorial
}

\section{Thoracic outlet syndrome: An uncommon (even rare) condition trying to find a home in industrial injuries}

My 35 years of experience in musculoskeletal diagnosis and treatment has left me with only a half-dozen or so cases of neurogenic thoracic outlet syndrome (TOS).

Why then are there pockets of frequent operations for this rare diagnosis? Some would suggest that pain in an upper limb not otherwise accounted for is the reason, and, of course, an obliging surgeon.

It is not infrequently that I am asked to evaluate a patient electrodiagnostically who has continued pain in an upper limb following a CTS release, ulnar nerve transposition, or posterior interosseus nerve decompression, and the surgeon is scheduling a TOS exploration.

We know that vascular TOS is identified by vascular studies and neurogenic TOS by electrodiagnostic exam. Our problem, however, is that many specialists do not appreciate the neurophysiologic techniques for verification of this rare disorder.
In the old days, it was simply a reduced CMAP of the thenar muscle and a reduced SNAP of digit 5. Now, we are more sophisticated and require a C-8 spinal nerve stimulation, F-wave in the ulnar nerve, as well as the CMAP of the abductor pollicis brevis muscle, the SNAP of digit 5 , and the appropriate needle EMG muscle distribution of abnormalities.

Thoracic outlet syndrome is rare, and even if present, hardly ever requires surgery. In my experience, many of these individuals complaining of upper limb discomfort have muscle pain (fibromyalgia), rather than nerve entrapment syndromes.

My management is 'use it or lose it' - a shorthand way of saying 'home exercise is the best way (probably the only way)'.

Ernest W. Johnson, $M D$ 